\title{
Suction of splash after impact on dry quick sand
}

\author{
G. A. Caballero-Robledo - Kevin P. D. Kelly • \\ Tess A. M. Homan • Joost H. Weijs • \\ Devaraj van der Meer • Detlef Lohse
}

Received: 23 September 2011 / Published online: 20 March 2012

(C) The Author(s) 2012. This article is published with open access at Springerlink.com

\begin{abstract}
It is well known that a splash occurs when an object impacts at high velocity on a liquid's surface. If the impact is fast enough, surface tension and air pressure gradients pull the crown-shape splash all the way towards the axis of symmetry, making it to collapse and seal the surface. In this paper we show that splash and surface sealing are also observed in impacts on soft, dry sand. We observe influence of air pressure and grains size on the shape of the splash. By tracking individual grains using high-speed imaging we calculate their acceleration, which results from gravity and drag forces. Assuming friction drag parallel, and pressure drag perpendicular to the direction of motion of grains we estimate the friction and pressure drag contributions to the drag force. Our results support the idea that pressure drag from Bernoulli effect is at the origin of the surface seal.
\end{abstract}

Keywords Granular systems · Fluidized beds .

Porous media

\section{Introduction}

When flowing, a granular material is very similar to a liquid. But the resemblance is only partial. Care must be taken when

Electronic supplementary material The online version of this article (doi:10.1007/s10035-012-0326-3) contains supplementary material, which is available to authorized users.

G. A. Caballero-Robledo

CINVESTAV - Monterrey, Nuevo León, México

K. P. D. Kelly · T. A. M. Homan · J. H. Weijs ·

D. van der Meer · D. Lohse $(\varangle)$

Physics of Fluids, MESA+ Institute for Nanotechnology, University of Twente, P.O. Box 217, 7500 AE, Enschede, The Netherlands

e-mail: d.lohse@utwente.nl thinking of a granular material as a liquid since it can behave as a highly unusual one [1].

An interesting example of sand flowing like a liquid is observed when a heavy object impacts onto a deep bed of grains. If the grains are fine and the packing is loose, an object can easily penetrate and move deep into the bed. When this happens, an impressive series of events occur [2-4]: (i) Splash; (ii) Void collapse and jet formation; and (iii) Eruption.

When an object impacts on the surface of a liquid very similar events are observed [5-14]. In a liquid, besides the deep closure of the cavity due to hydrostatic pressure, under some circumstances the ejecta sheet above the surface is pulled towards the symmetry axis and closes the cavity. The origin of the pulling force is a suction effect due to the pressure drop in the wake of the sphere [6]. Pressure inside the cavity has been directly measured finding that just before surface seal its value is an order of magnitude smaller than what expected from Bernoulli, $\frac{1}{2} \rho v_{0}^{2}$, where $\rho$ is the air density and $v_{0}$ the sphere velocity at impact [7].

A suction effect on the splash also exists in impact experiments on sand [3]. This is an indication that ambient pressure plays an important role in impacts on grains as it does in liquids.

The impact and penetration of an object into a bed of fine loose grains is a very complex phenomenon since it involves the coupled flow of grains and a fluid, in this case air. Here we study the role of air in shaping a granular splash to learn more about the subtleties of the interaction between grains and fluid.

\section{Experiments}

The experimental set up is the same used and described in references $[3,15,16]$. It consists of a $1 \mathrm{~m}$ high, transparent, perspex container of $14 \times 14 \mathrm{~cm}^{2}$ cross section and is half 

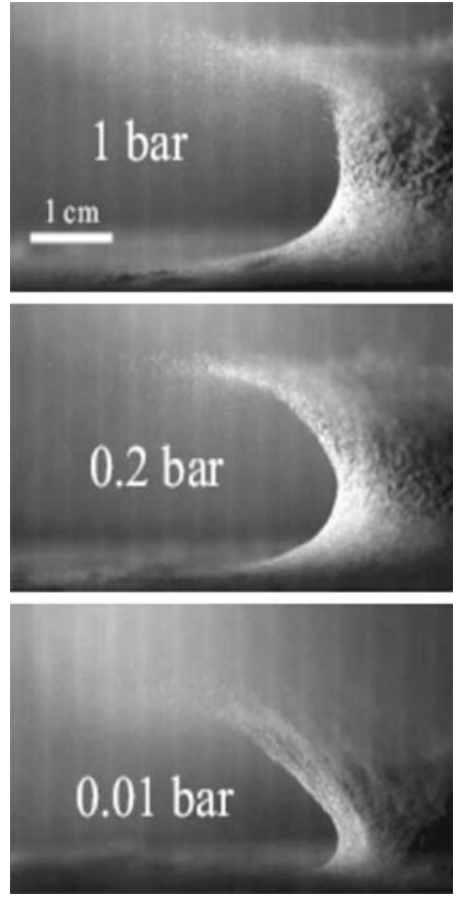

Fig. 1 Granular splash created by the impact of a steel sphere on a granular bed of fine, loose sand at different ambient pressures. The images show the splash $20 \mathrm{~ms}$ after impact. The bottom, right corner of the images coincide with the impact point, so only the left half of the splash is seen. A strong effect of the ambient air on the shape of the splash can be observed

filled with fine grains. Pressurized dry air is injected through a sintered plate to fluidize and expand the granular bed. Then the air flow is turned off and grains gently settle down in an extremely loose, static packing. Under these conditions the granular edifice is very fragile, unable to support any weight. Lohse et al. [17] called this state of sand "dry quick sand".

A vacuum pump connected to the top and bottom of the container allows to evacuate the air and reduce the ambient pressure. A pneumatic mechanism is used to release a steel sphere from an adjustable height $H$. The impact of the sphere on the free surface of the granular bed is imaged from one side with a high speed camera.

In the experiments presented in the next sections three different granular materials were used : (a) sand (nonspherical grains with sizes from 10 to $100 \mu \mathrm{m}$ ), and spherical glass beads whose sizes are in the range of (b) $40-70 \mu \mathrm{m}$ and (c) $70-110 \mu \mathrm{m}$. The projectile was a steel sphere of diameter $2 R=1.6 \mathrm{~cm}$ and mass $m=16.5 \mathrm{~g}$, released from rest to impact the surface at $3 \mathrm{~m} / \mathrm{s}$.

When the impact experiment is performed at low ambient pressures the shape of the splash is like a curved corona (see Fig. 1). Such shape can be fairly well reproduced by a model that considers most of the grains being ejected from the same position at a fixed angle but with different velocities [18]. After ejection, the grains follow a ballistic trajectory
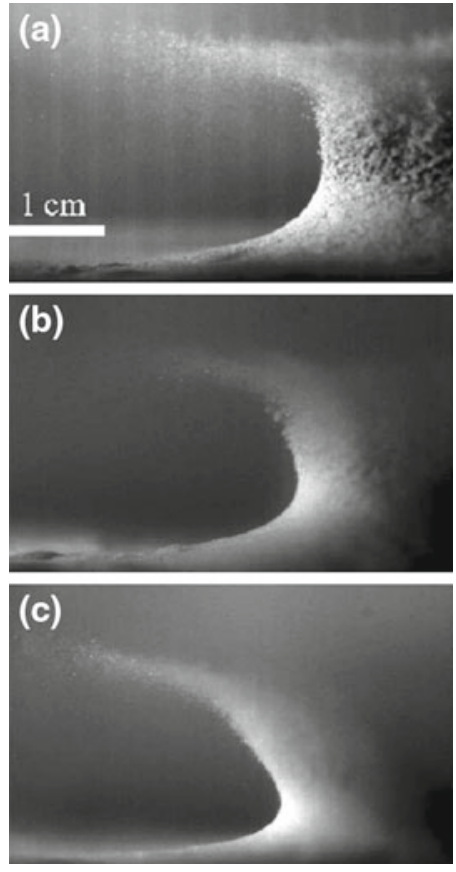

Fig. 2 Stills of splashes $20 \mathrm{~ms}$ after impact at atmospheric pressure, for (a) sand (grain sizes from 10 to $100 \mu \mathrm{m}$ ), and glass beads whose sizes are in the range of (b) $40-70 \mu \mathrm{m}$, and (c) $70-110 \mu \mathrm{m}$

and the angle that the edge of the corona makes with the horizontal remains constant in time. In our case, that angle is about 43 degrees. In contrast, at higher ambient pressure the ejected grains are pulled towards the center of the corona. This makes the walls of the corona to become more vertical (see Fig. 1).

\section{Effect of grain size}

The size of the grains that compose the granular bed has a great influence on the shape of the splash. Two different impact experiments performed under the same conditions, except for the size of the grains, create the same air flow outside the granular bed. However, it is expected to see a smaller deviation from a ballistic trajectory for the big grains since the friction and pressure drag scale at most with the square of the radius of the grains, while the mass scales with the cube of the radius.

Figure 2 shows the splash $20 \mathrm{~ms}$ after impact of three different experiments done under the same conditions but varying the size of the grains of the granular bed. Indeed, the deformation of the splash due to suction of grains into the cavity formed by the impacting ball is less important for bigger grains.

Figure 2 has other interesting features. When comparing images (a) and (b) one would expect the same shape of the splash since the size of the grains are similar: in (a) sand grain 
sizes go from 10 to $100 \mu \mathrm{m}$, while in (b) glass beads sizes are between 40 and $70 \mu \mathrm{m}$. But even if the sizes of grains in both cases are similar, the wall of the glass beads splash is less vertical than the sand splash. One possible explanation for this observation is that both materials behave differently when grains fly very close together, forming different kind of clusters. The origin of the formation of clusters in grains flying close together was recently explained by Royer et al. [19]: under these conditions, minute cohesive forces that arise from van der Waals forces and capillary bridges between nanometer-scale surface asperities are strong enough to cluster together the grains. Therefore, (small) changes in the shape and composition of the grains should lead to different cluster formation properties. Careful analysis of images (a) and (b) in Fig. 2 reveals that glass beads form bigger clusters than sand. Big clusters would behave like big grains regarding drag, thus reducing the effect of suction.

Another interesting feature found in Fig. 2 is the peculiar structure of the shape of the corona. In the three cases the structure of the splash can be thought as formed by three sections: the bottom part that goes from the bed surface until the height of the minimum cross sectional radius; then, there are two sections defined by straight walls, the lower one being less vertical than the one at the top of the splash. This observation, together with the fact that in all cases the splash height is the same, and the grains at the tip of the splash seem to be at the same distance from ejection point for the same time, suggest that the suction force acts on grains only for a limited time interval. That is to say, when the first grains are ejected, the cavity has not been formed yet and the pressure gradient has not built up either. So these grains fly almost ballistically, affected only by friction drag. At a later stage, the cavity is formed and the pressure gradient acts on grains, but the first grains ejected are already too far to be pulled towards the cavity. These grains form the top structure of the splash. The grains ejected when the pressure gradient is already present are deflected from the ballistic trajectory towards the cavity, forming the intermediate section of the splash with a more vertical wall. Finally, the cavity closes and all the grains continue to fly ballistically. We will show in the following sections that, indeed, the pressure drag stops being significant between 20 and $30 \mathrm{~ms}$ after impact, which coincides with the time at which the cavity begins to close [15].

\section{Particle tracking}

Impacts and splashes were recorded with a high speed camera at a rate of $10^{4}$ and $3 \times 10^{3}$ images per second for sand and for glass beads respectively. From these movies it is possible to track the position of individual grains as a function of time and calculate their velocity and acceleration by numerically

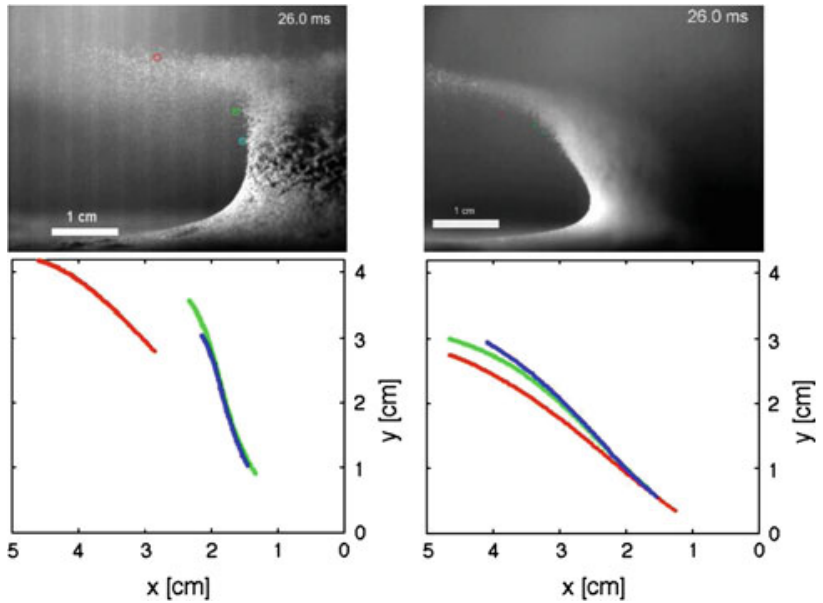

Fig. 3 (Color online) Granular splash at atmospheric pressure for sand (left) and glass beads with sizes between 70 to $110 \mu \mathrm{m}$ (right). From these kind of images it is possible to track the trajectory of individual grains. For each experiment three grains were tracked: a fast (red), a medium (green), and a slow one (blue). The origin of the reference system defined in the bottom curves is at the impact point

differentiating these trajectories. Figure 3 shows two frames of such high speed movies $26 \mathrm{~ms}$ after impact on sand and glass beads $(70-110 \mu \mathrm{m})$ at atmospheric pressure. For each movie three individual grains were identified whose positions could be clearly distinguished on each frame of the movie. A computational program was created that allows to go from frame to frame of the movie, make click on top of the tracked grain, and register the coordinates of its position on the image. The grains tracked are approximately of the size of one pixel in the images. So the uncertainty area determining the position of grains is a square of $3 \times 3$ pixels. In the case of sand one pixel corresponds to $124 \times 124 \mu \mathrm{m}^{2}$ so the uncertainty area is $372 \times 372 \mu \mathrm{m}^{2}$. For glass beads one pixel corresponds to $52 \times 52 \mu \mathrm{m}^{2}$ and the uncertainty area is $156 \times 156 \mu \mathrm{m}^{2}$. The grains tracked are circled with different colors in Fig. 3. In the same figure the whole trajectories of these grains are displayed. The whole movies with the tracked particles that correspond to the experiments presented in Fig. 3 are available on the Online Resource 1 for sand and Online Resource 2 for glass beads.

The first remarkable thing of the trajectories shown in Fig. 3 is that they deviate strongly from a simple ballistic trajectory. Another important feature is that the force responsible for this deviation seems to have an important component in the direction perpendicular to the velocity of the particles. This is interesting because a force perpendicular to the direction of movement of the grain is likely to be mainly caused by pressure drag and not friction drag. So, a calculation of the perpendicular force gives an estimation of the pressure drag.

Having obtained the position as a function of time of some individual grains from high speed movies we can estimate the 

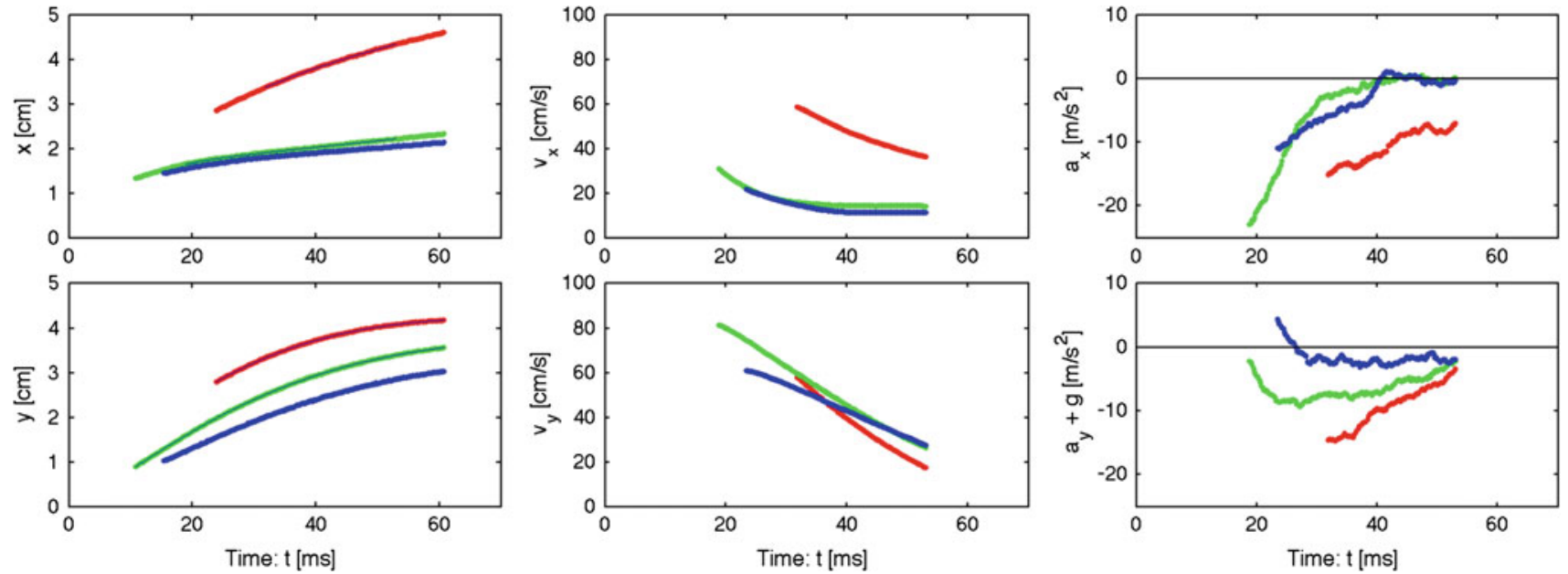

Fig. 4 (Color online) The vertical (top row) and horizontal (bottom row) velocities (center) and accelerations (right) are obtained by numerically differentiating the position of the grains as a function of time

(left). The data shown here correspond to the particles tracked in the sand splash movie at atmospheric pressure (see Fig. 3). In the top right figure the acceleration of gravity has been subtracted from the data

velocity and acceleration by numerically differentiating the data. Figure 4 shows the position, velocity and acceleration for the vertical (top row) and horizontal (bottom row) components of the trajectories of the three grains tracked after the impact in sand at atmospheric pressure.

The way in which the derivatives are calculated at a given point of the trajectory is by fitting a second order polynomial to a group of 80 (40) data points for sand (glass beads) around the desired point. The first and second derivatives of the fitted polynomial are taken as the derivatives of the trajectory at that point. Therefore, derivatives for the first and last 40 (20) data points of the trajectories are not presented since they would not be reliable. The points considered for the derivative at each point represent a time interval of $8 \mathrm{~ms}$ for sand and $13 \mathrm{~ms}$ for glass beads. It turns out that for smooth curves like those in Fig. 3 this kind of differentiation is more effective than a Savitzky-Golay method [20]. The uncertainty on the magnitude of the total acceleration calculated in this way is of $\pm 5 \mathrm{~m} / \mathrm{s}^{2}$. Note that the reference system is defined in Fig. 3 with the origin fixed at the impact point. Therefore, a negative vertical acceleration results from a force acting downwards while in the horizontal direction, a negative acceleration in this quadrant of the plane results from a force acting towards the axis of symmetry of the cavity. In the data presented in Fig. 4 the acceleration of gravity ( $-g$, since it acts downwards) is subtracted from the total vertical acceleration measured.

In this work we focus our attention on the analysis of trajectories of experiments performed at atmospheric pressure because only under these conditions are forces on particles strong enough to be detected by our estimation method. Besides, we do not analyze impacts on the small glass beads $(40-70 \mu \mathrm{m})$ because it is difficult to identify individual particles in the movies due to strong clustering present in this case. We are currently working on improving our tracking method to be able to systematically study the forces on grains at different pressures.

The total force acting on a grain of mass $m$ after ejection is $m \mathbf{a}=m \mathbf{g}+\mathbf{F}_{\text {Drag }}$, where $\mathbf{g}$ is the acceleration of gravity and $\mathbf{F}_{\text {Drag }}$ is the drag force, which can be decomposed in a friction drag and a pressure drag, $\mathbf{F}_{\text {Drag }}=\mathbf{F}_{\text {friction }}+\mathbf{F}_{\text {pressure }}$. Here, $\mathbf{F}_{\text {friction }}$ depends on the Reynolds number which is defined as $R e=\rho R_{\text {grain }} U / \eta$. In this definition $R_{\text {grain }}$ is the radius of the grain, $\eta$ is the dynamic viscosity of air, which is independent of the ambient pressure, $\mathbf{U}=\mathbf{v}_{\text {grain }}-\mathbf{v}_{\text {wind }}$, with $\mathbf{v}_{\text {wind }}$ the velocity of the air near the grain, and $\rho$ is the density of air at standard conditions. It turns out that the individual grains that we were able to track were discernible because they are particularly big grains, so they fly slightly out of the bulk during the splash. It is difficult to precisely determine the size of the tracked grains from the movies, but given the resolution of the images $\left(124 \times 124 \mu \mathrm{m}^{2} /\right.$ pixel for the sand experiment and $52 \times 52 \mu \mathrm{m}^{2} /$ pixel for the glass beads) we can roughly estimate $R_{\text {grain }} \sim 60 \mu \mathrm{m}$.

If the Reynolds number is calculated for grains immersed in a flow of air at atmospheric pressure, where the velocity of the flow is of the order of the impact velocity of the ball, i.e., $3 \mathrm{~m} / \mathrm{s}$, it turns out that the highest $R e$ associated to the dynamics of the grains in the splash is of the order of 20. This means that the friction drag is well described by Oseen's approximation, where the force depends on the ambient pressure through the density of air that appears in the Reynolds number, and this dependence is linear.

An interesting feature of the friction drag force is that its direction is defined by the difference of the velocity of the grain and the wind velocity, $\mathbf{U}=\mathbf{v}_{\text {grain }}-\mathbf{v}_{\text {wind }}$. It is reasonable to expect the wind velocity to be mainly directed towards the cavity, so for grains close to the ejection point 

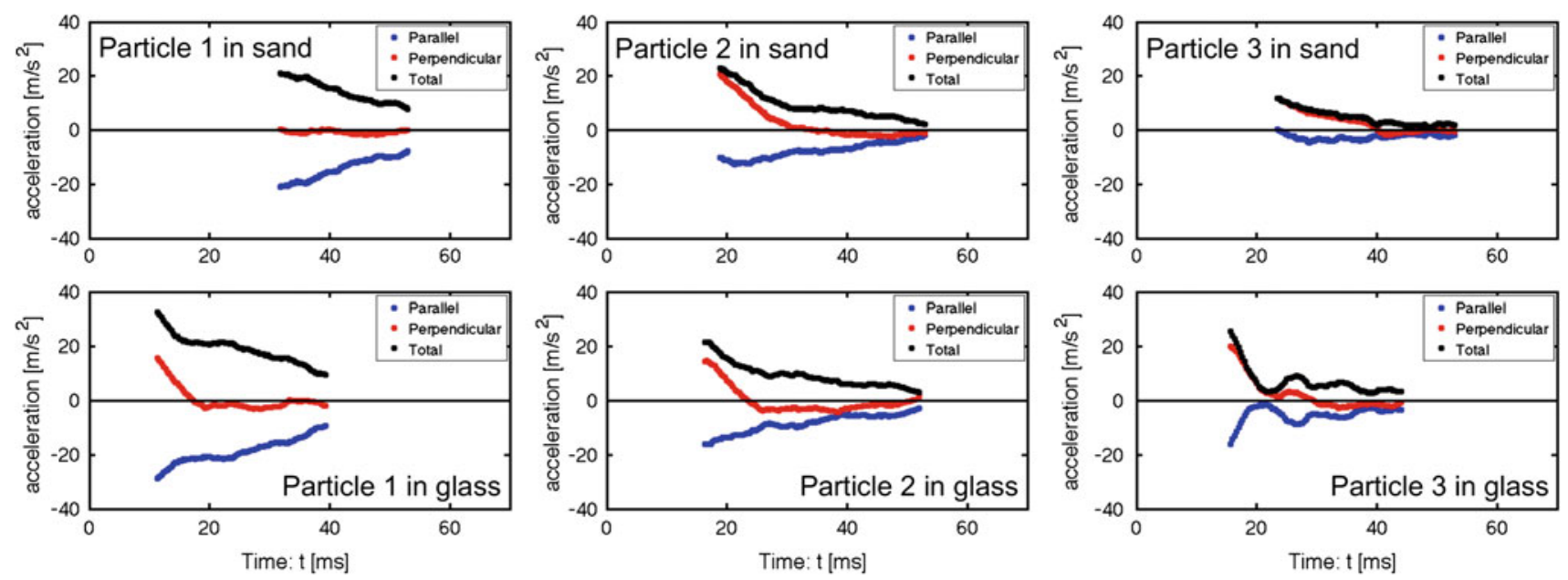

Fig. 5 (Color online) Acceleration of tracked particles for splashes at atmospheric pressure for sand (top row) and glass beads (bottom row). The data corresponding to particles one to three are ordered from left to right in each row. Black lines represent the total acceleration of the grains (excluding gravity), while the red and blue lines correspond respectively to the acceleration in the directions perpendicular and parallel to the instantaneous movement of the particles. These results are obtained from data like the one presented in Fig. 4 the wind velocity will closely oppose the direction of their own movement. Thus, the direction of the friction drag would be mainly directed in a direction parallel to the movement of the grain.

In contrast to friction drag, pressure drag is expected to be directed mainly in the direction perpendicular to the movement of the grains in the splash. Inside the splash the air flow velocity would be very strong, close to the velocity of the penetrating ball, while outside the splash the air flow would be almost zero. This big variation in the velocity of air in such a small region would build up strong pressure gradients due to the Bernoulli effect. Moreover, the force on grains due to these pressure gradients would be directed in the direction perpendicular to the movement of the grains.

We can estimate the order of magnitude of the pressure drag due to Bernoulli effect in a extreme case of a grain flying in a region where on one side it finds the air moving at the velocity of impact of the ball, $v_{\text {ball }}$, and on the other side the air is quiescent. The pressure in the quiescent part would be the ambient pressure $p_{0}$, while the pressure in the fast flow part $p_{B}$ would be given by the Bernoulli relation $\frac{1}{2} \rho v_{\text {ball }}^{2}+p_{B}(t)=p_{0}$. Thus, the pressure difference between the two regions would be $\Delta p=p_{0}-p_{B}=1 / 2 \rho v_{b a l l}^{2}$, and the pressure drag force would be $F_{\text {pressure }}=\Delta p \pi R_{\text {grain }}^{2}=$ $\frac{1}{2} \rho v_{\text {ball }}^{2} \pi R_{\text {grain }}^{2}$. Note that the dependence of this force on ambient pressure is linear. From this expression one can obtain the acceleration on the grain due to the pressure gradient $a_{\text {pressure }}=(3 / 8)\left(\rho / \rho_{\text {grain }}\right)\left(v_{\text {ball }}^{2} / R_{\text {grain }}\right)$, where $\rho / \rho_{\text {grain }}$ is the ratio of the air density and the density of the grain. For the case of an impact at $v_{\text {ball }} \approx 3 \mathrm{~m} / \mathrm{s}$, $R_{\text {grain }} \sim 60 \mu \mathrm{m}$, and $\rho_{\text {grain }} \approx 2.4 \mathrm{~g} / \mathrm{cm}^{3}$, the acceleration from pressure gradients would be $a_{\text {pressure }} \sim 30 \mathrm{~m} / \mathrm{s}^{2}$.
Now that we have an estimation of the expected pressure drag, we can compare it with the perpendicular component of the acceleration measured on the tracked particles. Figure 5 shows the acceleration of the tracked particles decomposed in the directions parallel (blue) and perpendicular (red) with respect to the instantaneous movement of the particle. In these plots, a positive perpendicular acceleration corresponds to the particle being accelerated to the right side with respect to its direction of motion, while a negative parallel acceleration means an acceleration in the direction opposite to the motion of the particle.

It is interesting to see in Fig. 5 that the perpendicular acceleration is of the order of magnitude of the above estimation of $a_{\text {pressure }}$. It is also noteworthy that this perpendicular acceleration is only significant for times smaller than $30 \mathrm{~ms}$. This time is consistent with the moment at which the cavity inside the bed stops growing due to its hydrostatically driven collapse (see figure 4 on reference [15]). When the cavity inside the bed stops growing, the flow of air into the cavity also stops, and the Bernoulli pressure gradients disappear, together with the perpendicular acceleration of particles.

The perpendicular acceleration is responsible for the deformation of the splash with respect to the low pressure shape (see Fig. 1). Moreover, the shape of the splash being very sensitive to the ambient pressure is consistent with the linear relation between the Bernoulli acceleration and density of air. This supports the idea that the Bernoulli effect is at the origin of the surface seal in granular impact experiments. However, a better tracking method is needed to analyze experiments at lower pressures and confirm its linear relation to acceleration. 
Summing up our observations we can propose the mechanism by which the shape of the splash is determined after an impact in a granular bed: (1) the ball impacts the bed surface ejecting the first grains of the splash; (2) the ball continues the penetration and ejection of grains until it is completely inside the granular bed; (3) the penetration continues and a cavity is formed behind the ball; (4) air is sucked into the cavity at high velocity building up strong pressure gradients in the neighbourhood of the cavity entrance; (5) the first grains ejected are far from the cavity so they are not affected by the pressure gradients, but grains ejected later are pulled towards the symmetry axis of the cavity; (6) the cavity begins to collapse and the air flows into the cavity less rapidly, reducing the pressure gradients; (7) grains in the splash continue flying almost ballistically, affected only by low Reynolds friction drag; (8) the splash evolves growing in size presenting a characteristic three part structure formed by the different group of grains ejected at different times; (9) the splash disappears by falling down.

\section{Conclusions}

In conclusion, we have studied the suction by ambient air of the splash which occurs after a heavy sphere impacts on dry quick sand. For large enough impact velocity, the Bernoulli effect of the air in the wake of the falling sphere leads to a surface seal of the splash. This study is another example of fluid-like behavior of dry quick sand in which air plays a crucial role: Without or with little ambient gas, there is no surface seal.

The quantitative analysis of the air effect is complicated by the fact that the influence of the ambient gas is threefold: First, there is an effect on the sand bed itself, which strongly influences the drag the ball experiences inside the sand and therefore its trajectory, second, on the splash, leading to a surface seal thanks to the Bernoulli effect, and third, on individual grains, which experience air friction drag. Disentangling these three different effects of the ambient air has turned out to be more complicated than anticipated, and clearly more quantitative studies with better visualization and grain-tracking techniques and a larger variation of the control parameters will have to be performed in the future.

Open Access This article is distributed under the terms of the Creative Commons Attribution License which permits any use, distribution, and reproduction in any medium, provided the original author(s) and the source are credited.

\section{References}

1. Jaeger, H.M., Nagel, S.R., Behringer, R.P.: Granular solids, liquids, and gases. Rev. Mod. Phys. 68(4), 1259-1273 (1996)

2. Thoroddsen, S.T., Shen, A.Q.: Granular jets. Phys. Fluids 13, 4-6 (2001)

3. Lohse, D., Bergmann, R., Mikkelsen, R., Zeilstra, C., van der, D.M., Versluis, M., van der, K.W., van der, M.H., Kuipers, H.: Impact on soft sand: void collapse and jet formation. Phys. Rev. Lett. 93(19), 198003 (2004)

4. Mikkelsen, R., Versluis, M., Koene, E., Bruggert, G.W., van der Meer, D., van der Weele, K., Lohse, D.: Granular eruptions: void collapse and jet formation. Phys. Fluids 14, S14 (2002)

5. Worthington, A.M., Cole, R.S.: Impact with a liquid surface studied by the aid of instantaneous photography. Paper II. Philos. Trans. Royal Soc. Lond. Ser. A Contain. Pap. Math. Phys. Character 194, 175-199 (1900)

6. Gilbarg, D., Anderson, R.A.: Influence of atmospheric pressure on the phenomena accompanying the entry of spheres into water. J. Appl. Phys. 19(2), 127-139 (1948)

7. Abelson, H.I.: Pressure measurements in the water-entry cavity. J. Fluid Mech. 44, 129-144 (1970)

8. Bergmann, R., van der Meer, D., Stijnman, M., Sandtke, M., Prosperetti, A., Lohse, D.: Giant bubble pinch-off. Phys. Rev. Lett. 96(15), 154505 (2006)

9. Duclaux, V., Caillé, F., Duez, C., Ybert, C., Bocquet, L., Canet, C.: Dynamics of transient cavities. J. Fluid Mech. 591, 1-19 (2007)

10. Gekle, S., Gordillo, J.M., van der Meer, D., Lohse, D.: Highspeed jet formation after solid object impact. Phys. Rev. Lett. 102(3), 034502 (2009)

11. Lee, M., Longoria, R.G., Wilson, D.E.: Cavity dynamics in highspeed water entry. Phys. Fluids 9(3), 540-550 (1997)

12. May, A.: Vertical entry of missile into water. J. Appl. Phys. 23(12), 1362-1372 (1952)

13. Glasheen, J.W., McMahon, T.A.: Vertical water entry of disks at low Froude numbers. Phys. Fluids 8(8), 2078-2083 (1996)

14. Duez, C., Ybert, C., Clanet, C., Bocquet, L.: Making a splash with water repellency. Nat. Phys. 3(3), 180-183 (2007)

15. Caballero, G., Bergmann, R., van der Meer, D., Prosperetti, A., Lohse, D.: Role of air in granular jet formation. Phys. Rev. Lett. 99(1), 018001 (2007)

16. von Kann, S., Joubaud, S., Caballero-Robledo, G.A., Lohse, D., van der Meer, D.: Effect of finite container size on granular jet formation. Phys. Rev. E 81(4), 041306 (2010)

17. Lohse, D., Rauhe, R., Bergmann, R., van der Meer, D.: Creating a dry variety of quicksand. Nature 432, 689 (2004)

18. Deboeuf, S., Gondret, P., Rabaud, M.: Dynamics of grain ejection by sphere impact on a granular bed. Phys. Rev. E 79(4), 041306 (2009)

19. Royer, J.R., Evans, D.J., Oyarte, L., Guo, Q., Kapit, E., Mobius, M.E., Waitukaitis, S.R., Jaeger, H.M.: High-speed tracking of rupture and clustering in freely falling granular streams. Nature 459(7250), 1110-1113 (2009)

20. Press, W.H., Teukolsky, S.A., Vetterling, W.T., Flannery, B.P.: Numerical Recipies: the Art of Scientific Computing. 3rd edn. Cambridge University Press, New York (2007) 